INPLASY

PROTOCOL

To cite: Zhang et al. Effect of Different Physical Activity Training Methods on Epilepsy: A protocol for systematic review and meta-analysis. Inplasy protocol 202220070. doi:

10.37766/inplasy2022.2.0070

Received: 18 February 2022

Published: 19 February 2022

Corresponding author:

Chen qi Zhang

zcq4869@163.com

Author Affiliation:

Chengdu BOE Hospital.

Support: grant no. 2016C001.

Review Stage at time of this submission: Preliminary

searches.

Conflicts of interest:

None declared.

\section{Effect of Different Physical Activity Training Methods on Epilepsy: A protocol for systematic review and meta-analysis}

Zhang, CQ1; Li, HY2; Wan, Y3; Bai, XY4; Gan, L5; Sun, HB6.

Review question / Objective: We aimed to provide guidance for selecting the optimal modality of physical exercise for PWE via a meta-analysis of available literature.

Condition being studied: Numerous studies have shown that exercise has been shown to be a physiological and psychological benefit for people with epilepsy. However, there are still many gaps in research concerning the effect of different physical exercise modalities (including aerobic exercise, resistance exercises or combined aerobic and resistance exercises) on the symptomatology, body composition, physical activity, physical fitness, cognitive function, mental emotion, and quality of life of individuals with epilepsy. we aim to compare the effects of different physical exercise programs on the symptomatology and health condition of individuals with epilepsy and provide guidance for selecting the optimal modality of physical exercise for patients with epilepsy via a meta-analysis of available literature.

INPLASY registration number: This protocol was registered with the International Platform of Registered Systematic Review and Meta-Analysis Protocols (INPLASY) on 19 February 2022 and was last updated on 19 February 2022 (registration number INPLASY202220070).

\section{INTRODUCTION}

Review question / Objective: we aimed to provide guidance for selecting the optimal modality of physical exercise for PWE via a meta-analysis of available literature.
Rationale: Epilepsy is a chronic disease of the CNS that affects individuals of all ages and has a worldwide distribution. As reported by the Lancet Neurology in 2019, there are currently 46 million patients with all-active patients with epilepsy(both idiopathic and secondary epilepsy ) 
globally. Prevalence of active epilepsy increased with age, with peaks at 5-9 years and at older than 80 years of age.Pharmacotherapy with anti-epileptic drugs(AEDs) remains the major treatment modality for epilepsy. Approximately $30 \%$ of patients with epilepsy continue to have seizures despite AEDs treatment. However, long-term use of these drugs may cause a variety of side effects and the most commonly reported adverse events across all drugs were drowsiness/fatigue, headache or migraine, gastrointestinal disturbances, dizziness/faintness and rash or skin disorders. Physical exercise is presented as a self-management strategy, can assist in reducing the frequency of seizures, higher self-esteem and social interaction, improving long-term physical well-being and optimize mental and reducing the disease burden for people living with epilepsy. However, for many years, people with epilepsy were advised not to engage in sports. Barriers prevent many PWE from exercising safely and confidently included a fear of injury, lack of social support, exercise-induced seizures (e.g., through overheating and/or high exercise intensity level) and have a negative effect on disease course. Numerous studies have shown that exercise has been shown to be a physiological and psychological benefit for people with epilepsy. However, there are still many gaps in research concerning the effect of different physical exercise modalities (including aerobic exercise, resistance exercises or combined aerobic and resistance exercises) on the symptomatology, body composition, physical activity, physical fitness, cognitive function, mental emotion, and quality of life of individuals with epilepsy.

Condition being studied: Numerous studies have shown that exercise has been shown to be a physiological and psychological benefit for people with epilepsy. However, there are still many gaps in research concerning the effect of different physical exercise modalities (including aerobic exercise, resistance exercises or combined aerobic and resistance exercises) on the symptomatology, body composition, physical activity, physical fitness, cognitive function, mental emotion, and quality of life of individuals with epilepsy. we aim to compare the effects of different physical exercise programs on the symptomatology and health condition of individuals with epilepsy and provide guidance for selecting the optimal modality of physical exercise for patients with epilepsy via a meta-analysis of available literature.

\section{METHODS}

Search strategy: We searched the following electronic bibliographic databases by using medical subject headings and term words: MEDLINE via PubMed, Cochrane Library, EBSCO, Embase, China National Knowledge(CNKI) and Wan fang databases (up toFebruary,2022).All the publications will be searched without any restriction of countries or article type. Reference list of all selected articles will independently screened to identify additional studies left out in the initial search.

Participant or population: The criteria for inclusion were as following : i)age $>12$ years ;ii) diagnosis of epilepsy based on International League Against Epilepsy criteria (ILAE2017) irrespective of their seizure type or epilepsy syndrome.

Intervention: The intervention group will be assigned with a supervised physical exercise program.

Comparator: The control group will be defined as patients which did not perform any regular physical exercise during the period where the study was conducted and just maintenance of usual activities

Study designs to be included: To decrease the risk of bias in individual studies, only studies that were randomized and controlled were included in this metaanalysis.

Eligibility criteria: Inclusion criteria: Randomized controlled blind/double-blind which reporting the interest outcomes (e.g., Depression, anxiety, psychiatric symptoms, 
sleep quality, overall quality of life) Exclusion criteria: (1) Observational studies, cohort studies, reviews, case report, letters, communication, editorials, lectures, conference abstracts; (2) Unclearly defined epilepsy; (3) Literatures with missing or repeated data.

Information sources: Electronic, and manual searching were combined to search all relevant literatures. Additionally, we also searched the bibliography of retrieved articles to obtain relevant articles.

Main outcome(s): The primary outcome measures were defined as any reduction in seizure frequency after implementation of the physical exercise.

Additional outcome(s): The additional outcome indicators were health variables based on comorbidities in epilepsy (including depression, anxiety, psychiatric symptoms, sleep quality, overall quality of life), changes in body composition and the incidences of adverse events associated to physical exercise.

Data management: Two researchers will independently extract data. Any disagreement will be resolved by discussion until consensus is reached or by consulting a third author.The following data will be extracted: the first author's name, publication date, rational, sample sizes, mean age, gender, details of participants (e.g., diagnostic criteria , epilepsy types or syndrome, AEDs, etc.), interventions (e.g., means and duration times of physical exercise), interested outcomes and AEs.

Quality assessment / Risk of bias analysis: The quality of each study will be appraised by using the Cochrane Risk of Bias assessment tool. The domains including the following 7 aspects, include random sequence generation, allocation concealment, blinding of the participants, blinding of outcome assessments, incomplete outcome data, and selective outcome reporting.

Strategy of data synthesis: Stata 16.0 software will be performed for statistical analyses . Standardized mean difference (SMD) with the $95 \% \mathrm{Cl}$ as an effect size was measured for continuous data. A dichotomous analysis with the risk ratios (RRs) and $95 \%$ confidence intervals (Cls) were calculated to compare differences between groups. The heterogeneity was assessed by the Cochran's $Q$ test and 12 test. If $\mathrm{P} 50 \%$, data were analyzed using a random-effects model; Otherwise, they were analyzed using a fixed-effects model. Two-tailed $\mathrm{P}<0.05$ was considered statistically significant.

Subgroup analysis: Had we had sufficient data, we would have carried out the subgroup analyses(e.g., duration times of physical exercise and types of physical exercise).

Sensitivity analysis: If necessary, a leaveone-out sensitivity analysis will be performed to evaluate the main trials demonstrating a substantial impact on the inter-study heterogeneity.we will conduct sensitivity analyses to assess the robustness of the results.

Language: None.

Country(ies) involved: China.

Keywords: Physical exercise; epilepsy; meta-analysis.

Dissemination plans: Ethics and dissemination Ethical approval are not required since it is secondary data analysis based on published literature.

Contributions of each author:

Author 1 - Chen qi Zhang.

Email: zcq4869@163.com

Author 2 - Hongyan Li.

Author 3 - Yong Wan.

Author 4 - Xueyang Bai.

Author 5 - Lu Gan.

Author 6 - Hongbin Sun. 